PROCEEDINGS OF THE

AMERICAN MATHEMATICAL SOCIETY

Volume 140, Number 11, November 2012, Pages 3677-3686

S 0002-9939(2012)11207-9

Article electronically published on February 29, 2012

\title{
ON LOCAL TRIGONAL FIBRATIONS
}

\author{
MIZUHO ISHIZAKA AND HIRO-O TOKUNAGA \\ (Communicated by Ted Chinburg)
}

\begin{abstract}
We show that certain degenerating hyperelliptic curves, called horizontal types, of genus $g$ appear as the central fiber of a local family of trigonal curves of genus $g$ by constructing such a family explicitly.
\end{abstract}

\section{INTRODUCTION}

Let $\varphi: \mathcal{C}=\left\{\mathcal{C}_{t}\right\}_{t \in \Delta_{\epsilon}} \rightarrow \Delta_{\epsilon}$ be a local family of curves over a small disc $\Delta_{\epsilon}:=$ $\{t \in \mathbb{C}|| t \mid<\epsilon\}$, where $\epsilon$ is a small positive real number, which means that $\mathcal{C}$ is a smooth surface, $\varphi$ is proper and surjective, all fibers of $\varphi$ are connected and $\varphi^{-1}(t)$ is smooth for $t \neq 0$. We call $\varphi: \mathcal{C} \rightarrow \Delta_{\epsilon}$ a local hyperelliptic (resp. trigonal) fibration of genus $g$ if $\mathcal{C}_{t}(t \neq 0)$ is a hyperelliptic (resp. trigonal) curve of genus $g$.

In 4, Chen and Tan gave two examples of local trigonal fibrations of genus 3 such that their central fibers, $\mathcal{C}_{0}$, are smooth hyperelliptic curves of genus 3 . A purpose of this paper is to show that any hyperelliptic curve of genus $g$ appears as the central fiber of a local trigonal fibration.

Let us explain our setting and question precisely. Let $B_{1}$ be a reduced divisor on $\mathbb{P}^{1} \times \Delta_{\epsilon}$ such that $B_{1}$ meets $\mathbb{P}^{1} \times\{t\}(t \neq 0)$ at $2 g+2$ distinct points transversely. In particular, all singularities of $B_{1}$ are in $\mathbb{P}^{1} \times\{0\}$. Let $f_{o}: W \rightarrow \mathbb{P}^{1} \times \Delta_{\epsilon}$ be the double cover with $\Delta_{f_{o}}=B_{1}$, where $\Delta_{f_{o}}$ denotes the branch locus of $f_{o}$, and let $\mu_{o}: \widetilde{W} \rightarrow W$ be the canonical resolution (see 7 for the canonical resolution). We consider a local hyperelliptic fibration of genus $g$ given by putting $\mathcal{C}=\widetilde{W}$ and $\varphi_{o}:=\operatorname{pr}_{2} \circ f_{o} \circ \mu_{o}$.

We say that

(i) $\varphi_{o}: \mathcal{C} \rightarrow \Delta_{\epsilon}$ is of horizontal type if $B_{1}$ does not contain $\mathbb{P}^{1} \times\{0\}$ as its irreducible component, and

(ii) $\varphi_{o}: \mathcal{C} \rightarrow \Delta_{\epsilon}$ is of non-horizontal type if $B_{1}$ contains $\mathbb{P}^{1} \times\{0\}$ as its irreducible component.

Note that we may assume that $\varphi_{o}$ is either horizontal or non-horizontal by taking $\epsilon(>0)$ small enough. Also any local hyperelliptic fibration is obtained as the relatively minimal model of $\varphi_{o}: \mathcal{C} \rightarrow \Delta_{\epsilon}$. We now formulate our question as follows:

Question 0.1. For an arbitrary local hyperelliptic fibration $\varphi_{0}: \mathcal{C} \rightarrow \Delta_{\epsilon}$, does there exist a local trigonal fibration $\varphi: \widetilde{\mathcal{C}} \rightarrow \Delta_{\epsilon}$ such that $\mathcal{C}_{0}=\widetilde{\mathcal{C}}_{0}$ ? In other words,

Received by the editors October 8, 2007 and, in revised form, December 16, 2010; March 17, 2011; and April 19, 2011.

2010 Mathematics Subject Classification. Primary 14H15; Secondary 14H30, 14H51.

Key words and phrases. Trigonal curve, triple cover.

(C)2012 American Mathematical Society Reverts to public domain 28 years from publication 
can the central fiber of any local hyperelliptic fibration appear as that of a certain local trigonal fibration?

In this paper, we give an answer to Question 0.1 in the case when $\varphi_{o}$ is of horizontal type.

Theorem 0.1. Question 0.1 is true for a local hyperelliptic fibration of horizontal type.

Since any hyperelliptic curve of genus $g$ appears as the centeral fiber of a local hyperelliptic fibration of horizontal type, i.e., a trivial fibration, we have

Corollary 0.1. Let $D$ be any hyperelliptic curve of genus $g$. There exists a local trigonal fibration $\widetilde{\varphi}: \widetilde{\mathcal{C}} \rightarrow \Delta_{\epsilon}$ such that $\widetilde{\mathcal{C}}_{0}=D$.

\section{Covers and the fundamental group}

Let $X$ and $M$ be a normal variety and a complex manifold, respectively. We call $X$ a (branched) cover of $M$ if there exists a finite surjective morphism $\pi: X \rightarrow M$. When needed, the covering morphism will be specified as a cover $\pi: X \rightarrow Y$. Let $B$ be a reduced divisor on $M$. The following facts are well-known:

- Choose a point $* \in M \backslash B$. Then the inclusion morphism $\iota: M \backslash B \rightarrow M$ induces an epimorphism $\iota_{*}: \pi_{1}(M \backslash B, *) \rightarrow \pi_{1}(M, *)$.

- Let $H$ be a subgroup of $\pi_{1}(M \backslash B, *)$. Then there exists an unramified cover $\pi_{H}: X_{o} \rightarrow M \backslash B$ over $M \backslash B$ with $\pi_{H}\left(X_{o}, \hat{*}\right) \cong H, \pi_{H}(\hat{*})=*$, such that $X_{o}$ can be extended over $M$ uniquely. We also denote the extended cover of $\pi_{H}: X_{0} \rightarrow M \backslash B$ by $\pi_{H}: X \rightarrow M$. Note that the branch locus $\Delta_{\pi_{H}}$ of $\pi_{H}$ is a subset of $B$ and $\operatorname{deg} \pi_{H}=\left[\pi_{1}(M \backslash B, *): H\right]$. Conversely, if there exists a branched cover $\pi: X \rightarrow M$ with $\operatorname{deg} \pi=n$, then there exists a subgroup $H_{\pi}$ of $\pi_{1}\left(M \backslash \Delta_{\pi}, *\right)$ of index $n$.

- Let $H$ be a normal subgroup of $\pi_{1}(M \backslash B, *)$. Then there exists a Galois cover $\pi_{H}: X \rightarrow M$ with $\operatorname{Aut}_{M}(X) \cong \pi_{1}(M \backslash B, *) / H$.

For the first statement, see [9], for example, and for the last two statements, see 6], EXPOSE XII, for example.

Let $G$ be a finite group. We call a Galois cover with $\operatorname{Aut}_{M}(X) \cong G$ a $G$-cover.

The following lemma is fundamental throughout this article. Note that Tan proved a similar theorem ([11, Corollary 2.3) using algebro-geometric methods.

Lemma 1.1. Let $M$ and $B$ be as above. Let $D_{6}$ be the dihedral group of order 6 , which we describe by $\left\langle\sigma, \tau \mid \sigma^{2}=\tau^{3}=(\sigma \tau)^{2}=1\right\rangle$. If there exists a non-Galois triple cover $\pi: X \rightarrow M$ with $\Delta_{\pi}=B$, then there exists a $D_{6}$-cover $\hat{\pi}: \hat{X} \rightarrow M$, with $\Delta_{\hat{\pi}}=B$. Conversely, if there exists a $D_{6}$-cover $\hat{\pi}: \widehat{X} \rightarrow M$ with $\Delta_{\hat{\pi}}=B$, then the quotient surface $X:=\widehat{X} /\langle\sigma\rangle$ by $\sigma$ admits a non-Galois triple cover $\pi: X \rightarrow M$ with $\Delta_{\pi}=B$.

Proof. Let $\pi: X \rightarrow M$ be a given non-Galois triple cover of $M$ as above. Then there exists a corresponding subgroup $H$ of $\pi_{1}(M \backslash B, *)$ with $\pi=\pi_{H}$ and $\left[\pi_{1}(M \backslash B, *)\right.$ : $H]=3$. By considering the permutation action over the set of all left cosets of $H$, we have a homomorphism from $\pi_{1}(M \backslash B, *)$ to the symmetric group $\mathfrak{S}_{3}$ of three letters and its image is a transitive subgroup of $\mathfrak{S}_{3} \cong D_{6}$ (see [10, Chapter I, $\S 7]$ ). Since $\pi$ is not Galois, $H$ is not normal. This implies that there exists an 
epimorphism $\pi_{1}(M \backslash B, *) \rightarrow D_{6}$ such that $K:=\operatorname{ker}\left(\pi_{1}(M \backslash B, *) \rightarrow D_{6}\right) \subset$ $H$. The $D_{6}$-cover corresponding to $K$ satisfies the desired property. Conversely, suppose that there exists a $D_{6}$-cover $\varpi: \mathcal{X} \rightarrow M$ with $\Delta_{\varpi}=B$. As we have an epimorphism $\pi_{1}(M \backslash B, *) \rightarrow D_{6}$, there exists a subgroup, $H$, of $\pi_{1}(M \backslash B, *)$ such that $\left[\pi_{1}(M \backslash B, *): H\right]=3$ and $H$ contains $\operatorname{ker}\left(\pi_{1}(M \backslash B, *) \rightarrow D_{6}\right)$. The triple cover corresponding to $H$ is the desired one. As for the equality $\Delta_{\varpi}=\Delta_{\pi_{H}}$, see [12.

Remark 1.1. Let $\pi: X \rightarrow M$ be as in Lemma 1.1. There exists a double cover corresponding to the preimage of the subroup of order 3 in $D_{6}$. We denote it by $\beta_{1}(\pi): D(X / M) \rightarrow M$. Note that $\Delta_{\beta_{1}(\pi)} \subseteq \Delta_{\pi}$.

\section{Proof of Theorem 0.1}

2.1. Settings. Throughout this section, we always assume that

$(*)$ a local hyperelliptic fibration is of horizontal type.

Let $B_{1}$ be the branch locus of $f_{o}: W \rightarrow \mathbb{P}^{1} \times \Delta_{\epsilon}$ as in the Introduction. Let $B_{o}$ be a double section of $\mathrm{pr}_{2}: \mathbb{P}^{1} \times \Delta_{\epsilon} \rightarrow \Delta_{\epsilon}$ such that

- $B_{o}$ is smooth,

- $\left.\operatorname{pr}_{2}\right|_{B_{o}}: B_{o} \rightarrow \Delta_{\epsilon}$ has a unique ramification point over $0 \in \Delta_{\epsilon}$, and

- $B_{o} \cap B_{1}=\emptyset$.

Put $B:=B_{o}+B_{1}$ and $G_{B}:=\pi_{1}\left(\mathbb{P}^{1} \times \Delta_{\epsilon} \backslash B, *\right)$. In order to study non-Galois triple covers with branch locus $B$, by Lemma 1.1, we need to know the description of $G_{B}$ and its normal subgroup $K$ with $G_{B} / K \cong D_{6}$.

2.2. A description of $G_{B}$. We describe $G_{B}$ via generators and their relations. The method used here is well-known in computing the fundamental group of the complements of plane curves via the so-called "Zariski-van Kampen method". We refer to [1, 2, 5, 9] and use results there freely.

Choose a point $t_{o}$ in $\Delta_{\epsilon}^{*}:=\Delta_{\epsilon} \backslash\{0\}$. Put $\mathbb{P}_{t_{o}}^{1}:=\mathbb{P}^{1} \times\left\{t_{o}\right\}$ and $\mathbf{Y}:=\mathbb{P}_{t_{o}}^{1} \cap B$. Let $\eta$ be a loop given by $\left\{\left|t_{o}\right| \exp (2 \pi i \theta) \mid 0 \leq \theta \leq 1\right\}$. Note that $\pi_{1}\left(\Delta_{\epsilon}^{*}, t_{o}\right) \cong\langle\eta\rangle \cong \mathbb{Z}$. Choose a point $b=\left(\hat{t}_{o}, t_{o}\right) \in\left(\mathbb{P}^{1} \times \Delta_{\epsilon}^{*}\right) \backslash B$ with $\left(\left\{\hat{t}_{o}\right\} \times \Delta_{\epsilon}\right) \cap B=\emptyset$ and a geometric basis $\gamma_{1}, \gamma_{2}, \ldots, \gamma_{2 g+4}$ of $\pi_{1}\left(\mathbb{P}_{t_{o}}^{1} \backslash \mathbf{Y}, \hat{t}_{o}\right)$ (see [1, Definition 1.13] for the definition of a geometric basis). One can define a right action of $\pi_{1}\left(\Delta_{\epsilon}^{*}, t_{o}\right)$ on $\pi_{1}\left(\mathbb{P}_{t_{o}}^{1} \backslash \mathbf{Y}, \hat{t}_{o}\right)$, and $G_{B}$ is described through this action as follows:

$$
G_{B} \cong\left\langle\gamma_{1}, \ldots, \gamma_{2 g+4} \mid \gamma_{i}^{\eta}=\gamma_{i}(i=1, \ldots, 2 g+4), \gamma_{1} \ldots \gamma_{2 g+4}=1\right\rangle .
$$

We may assume that and

$\gamma_{1}, \ldots, \gamma_{2 g+2}$ are meridians for the points in $\mathbb{P}_{t_{o}}^{1} \cap B_{1}$,

$\gamma_{2 g+3}$ and $\gamma_{2 g+4}$ are meridians for $\mathbb{P}_{t_{o}}^{1} \cap B_{o}$.

Under these circumstances, we have

\section{Lemma 2.1.}

$G_{B} \cong\left\langle\gamma_{1}, \ldots, \gamma_{2 g+4} \mid \gamma_{i}^{\eta}=\gamma_{i}(i=1, \ldots, 2 g+2), \gamma_{2 g+3}=\gamma_{2 g+4}, \gamma_{1} \cdots \gamma_{2 g+4}=1\right\rangle$.

Let $H$ be the subgroup of $G_{B}$ generated by

$$
\gamma_{i}^{2}(i=1, \ldots, 2 g+4) \text { and } \gamma_{j} \gamma_{j+1}(j=1, \ldots, 2 g+3) .
$$

Let $f: S \rightarrow \mathbb{P}^{1} \times \Delta_{\epsilon}$ be a double cover with $\Delta_{f}=B$. 
Lemma 2.2. $H$ is the subgroup of $G_{B}$ corresponding to the double cover

$$
f^{\prime}:=\left.f\right|_{S \backslash f^{-1}(B)}: S \backslash f^{-1}(B) \rightarrow\left(\mathbb{P}^{1} \times \Delta_{\epsilon}\right) \backslash B .
$$

Proof. Put $\bar{\varphi}:=\operatorname{pr}_{2} \circ f$ and $S_{t_{o}}:=\bar{\varphi}^{-1}\left(t_{o}\right)$. Let $f_{t_{o}}: S_{t_{o}} \rightarrow \mathbb{P}_{t_{o}}^{1}$ be the restriction of $f$ to the fiber over $t_{o}$ and put $f_{t_{o}}^{\prime}:=\left.f_{t_{o}}\right|_{S_{t_{o}} \backslash f_{t_{o}}^{-1}(\mathbf{Y})}$. We have a commutative diagram:

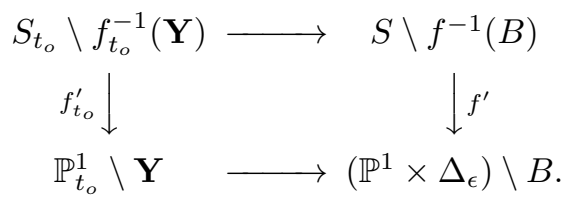

From the above diagram, we have a commutative diagram of groups:

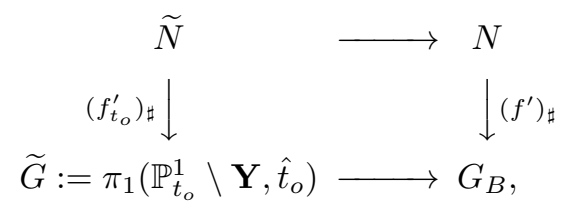

where $\tilde{N}$ and $N$ are the subgroups of index 2 corresponding to the double covers $f_{t_{o}}^{\prime}$ and $f^{\prime}$; i.e., $\widetilde{N}$ and $N$ can be considered as the fundamental groups of $S_{t_{o}} \backslash f_{t_{o}}^{-1}(\mathbf{Y})$ and $S \backslash f^{-1}(B)$, respectively. Since $S \backslash f^{-1}\left(B \cup \mathbb{P}^{1} \times\{0\}\right) \rightarrow \Delta_{\epsilon} \backslash\{0\}$ is a submersion, $S \backslash f^{-1}\left(B \cup \mathbb{P}^{1} \times\{0\}\right)$ is a fiber bundle over $\Delta_{\epsilon} \backslash\{0\}$ whose fiber is $S_{t_{o}} \backslash f_{t_{o}}^{-1}(\mathbf{Y})$. Hence we have an exact sequence

$$
\pi_{1}\left(S_{t_{o}} \backslash f_{t_{o}}^{-1}(\mathbf{Y})\right) \rightarrow \pi_{1}\left(S \backslash f^{-1}\left(B \cup\left(\mathbb{P}^{1} \times\{0\}\right)\right)\right) \rightarrow \pi_{1}\left(\Delta_{\epsilon} \backslash\{0\}\right) \rightarrow 1,
$$

where the base points of each fundamental group are appropriately chosen.

Now we can apply the argument of the proof of [9, Lemma 1.5, C]. We have

$$
\pi_{1}\left(S_{t_{o}} \backslash f_{t_{o}}^{-1}(\mathbf{Y})\right) \rightarrow \pi_{1}\left(S \backslash f^{-1}(B)\right) \rightarrow \pi_{1}\left(\Delta_{\epsilon}\right) \rightarrow 1 .
$$

Hence $\widetilde{N} \rightarrow N$ is surjective. As $\left[G_{B}: N\right]=2$, our statement follows from the claim below:

Claim. Let $\widetilde{H}$ be the subgroup of $\widetilde{G}$ generated by

$$
\gamma_{i}^{2}(i=1, \ldots, 2 g+4) \text { and } \gamma_{j} \gamma_{j+1}(j=1, \ldots, 2 g+3) .
$$

Then $\widetilde{H}=\widetilde{N}$.

Proof of Claim. Since the branch locus of $f_{t_{o}}: S_{t_{o}} \rightarrow \mathbb{P}_{t_{o}}^{1}$ is $\mathbf{Y}, \widetilde{H} \subset \widetilde{N}$. It is enough to show that $\widetilde{G}=\widetilde{H} \cup \gamma_{1}^{-1} \widetilde{H}$, i.e., $[\widetilde{G}: \widetilde{H}]=2$.

Step 1. $\gamma_{i}^{ \pm} \in \gamma_{1}^{-1} \widetilde{H}$.

We first note that $\gamma_{1}=\gamma_{1}^{-1} \gamma_{1}^{2} \in \gamma_{1}^{-1} \widetilde{H}$. Suppose that $\gamma_{i} \in \gamma_{1}^{-1} \widetilde{H}$ and put $\gamma_{i}=\gamma_{1}^{-1} h_{i}, h_{i} \in \widetilde{H}$. Then

$$
\gamma_{i+1}=\gamma_{i}^{-1} \gamma_{i} \gamma_{i+1}=\gamma_{i} \gamma_{i}^{-2}\left(\gamma_{i} \gamma_{i+1}\right)=\gamma_{1}^{-1} h_{i} \gamma_{i}^{-2}\left(\gamma_{i} \gamma_{i+1}\right) \in \gamma_{1}^{-1} \widetilde{H} .
$$

Thus $\gamma_{i} \in \gamma_{1}^{-1} \widetilde{H}$ for $i=1, \ldots, 2 g+4$. Also $\gamma_{i}^{-1}=\gamma_{i} \gamma_{i}^{-2} \in \gamma_{1}^{-1} \widetilde{H}$.

Step 2. Any element on $w \in \widetilde{G}$ is in either $\widetilde{H}$ or $\gamma_{1}^{-1} \widetilde{H}$.

Since

$$
\widetilde{G}=\left\langle\gamma_{1}, \ldots, \gamma_{2 g+4} \mid \gamma_{1} \gamma_{2} \cdot \ldots \cdot \gamma_{2 g+4}=1\right\rangle,
$$


we may assume that any $w \in \widetilde{G}$ is of the form

$$
w=w_{1} \cdot \ldots \cdot w_{M},
$$

where $w_{k}=\gamma_{i}$ or $\gamma_{i}^{-1}$ for some $i$.

Suppose that $M$ is even. As $w=\left(w_{1} w_{2}\right) \cdots\left(w_{i} w_{i+1}\right) \cdots\left(w_{M-1} w_{M}\right)$, it is enough to show that

$$
\gamma_{i}^{ \pm} \gamma_{j}^{ \pm}, \gamma_{i}^{ \pm} \gamma_{j}^{\mp} \in \widetilde{H}
$$

for any $i, j$. Since

$$
\gamma_{i}^{-1} \gamma_{j}^{-1}=\left(\gamma_{j} \gamma_{i}\right)^{-1}, \quad \gamma_{i}^{-1} \gamma_{j}=\gamma_{i}^{-2} \gamma_{i} \gamma_{j}, \quad \gamma_{i} \gamma_{j}^{-1}=\gamma_{i} \gamma_{j} \gamma_{j}^{-2},
$$

we only need to show that $\gamma_{i} \gamma_{j} \in \widetilde{H}$ for any $i, j$. There are three possibilities: namely, $(i) i=j,(i i) i<j$ and (iii) $i>j$, and we check each case separately.

The case $(i) . \gamma_{i} \gamma_{j}=\gamma_{i}^{2} \in \widetilde{H}$.

The case (ii). Since

$$
\gamma_{i} \gamma_{j}=\left(\gamma_{i} \gamma_{i+1}\right) \gamma_{i+1}^{-2}\left(\gamma_{i+1} \gamma_{i+2}\right) \cdot \ldots \cdot \gamma_{j-1}^{-2} \gamma_{j-1} \gamma_{j},
$$

$\gamma_{i} \gamma_{j} \in \widetilde{H}$

The case (iii). Since

$$
\gamma_{i} \gamma_{j}=\left(\gamma_{i} \gamma_{i-1}\right) \gamma_{i-1}^{-2}\left(\gamma_{i-1} \gamma_{i-2}\right) \cdot \ldots \cdot \gamma_{j+1}^{-2} \gamma_{j+1} \gamma_{j}
$$

and

$$
\gamma_{k+1} \gamma_{k}=\gamma_{k+1}^{2}\left(\gamma_{k+1}^{-1} \gamma_{k}^{-1}\right) \gamma_{k}^{2}=\gamma_{k+1}^{2}\left(\gamma_{k} \gamma_{k+1}\right)^{-1} \gamma_{k}^{2},
$$

we infer that $\gamma_{i} \gamma_{j} \in \widetilde{H}$. Thus $w \in \widetilde{H}$ when $M$ is even.

Suppose that $M$ is odd. In this case, $w$ is of the form $\gamma_{i} \widetilde{w}$ or $\gamma_{i}^{-1} \widetilde{w}$ for some $i$ and $\widetilde{w} \in \widetilde{H}$ by Case 1 . Since $\gamma_{i}^{ \pm} \in \gamma_{1}^{-1} \widetilde{H}$, we infer that $w \in \gamma_{1}^{-1} \widetilde{H}$.

From Step 1 and Step 2, we have the Claim and Lemma 2.2 follows.

2.3. Existence of a $D_{6}$-cover. In this section, we show the existence of a normal subgroup $K$ of $G_{B}$ such that (i) $K$ is a subgroup of $H,($ ii $) G_{B} / K \cong D_{6}$ and (iii) the $D_{6}$-cover corresponding to $K$ is branched along $B$ with ramification index 2 .

Let $\mu: \widetilde{S} \rightarrow S$ be the canonical resolution of the double cover $f: S \rightarrow \mathbb{P}^{1} \times \Delta_{\epsilon}$. Since singularities of $S$ are on those of $B$, we have $S \backslash f^{-1}(B) \cong \tilde{S} \backslash(f \circ \mu)^{-1}(B)$. Hence we have an epimorphism $\bar{\delta}: \pi_{1}\left(S \backslash f^{-1}(B), b_{+}\right) \rightarrow \pi_{1}\left(\widetilde{S}, b_{+}\right)$, where $b_{+}$is a point in $f^{-1}(b)$. In particular, we have an epimorphism $\delta: H \rightarrow H_{1}(\widetilde{S}, \mathbb{Z})$.

Lemma 2.3. For any $h \in H$, we have

$$
\delta\left(\gamma_{1}^{-1} h \gamma_{1}\right)=-\delta(h)=\delta\left(h^{-1}\right) .
$$

Proof. We first note that $\gamma_{i}^{2} \in \operatorname{ker} \delta$ for $i=1, \ldots, 2 g+4$. Since

$$
\gamma_{1}^{-1}\left(\gamma_{i} \gamma_{i+1}\right) \gamma_{1}=\left(\gamma_{i} \gamma_{1}\right)^{-1} \gamma_{i}^{2} \gamma_{i+1}^{2}\left(\gamma_{i} \gamma_{i+1}\right)^{-1}\left(\gamma_{i} \gamma_{1}\right) \text {, }
$$

we have

$$
\delta\left(\gamma_{1}^{-1}\left(\gamma_{i} \gamma_{i+1}\right) \gamma_{1}\right)=-\delta\left(\gamma_{i} \gamma_{i+1}\right) .
$$


Let $h$ be an arbitrary element in $H$ and suppose that $h$ is of the form $u_{1} \cdot \ldots \cdot u_{n}$, where $u_{i}(i=1, \ldots, n)$ are either $\left(\gamma_{j}^{2}\right)^{ \pm}$or $\left(\gamma_{l} \gamma_{l+1}\right)^{ \pm}$for some $j, l$. Then

$$
\begin{aligned}
\delta\left(\gamma_{1}^{-1} h \gamma_{1}\right) & =\sum_{i=1}^{n} \delta\left(\gamma_{1}^{-1} u_{i} \gamma_{1}\right) \\
& =-\sum_{i=1}^{n} \delta\left(u_{i}\right) \\
& =-\delta(h) .
\end{aligned}
$$

Let $A$ be a subgroup of $H_{1}(\widetilde{S}, \mathbb{Z})$ and put $K_{A}:=\delta^{-1}(A)$. We have

Lemma 2.4. $K_{A}$ is a normal subgroup of $G_{B}$.

Proof. Let $k$ be an arbitrary element of $K_{A}$. By Lemma 2.3, $\delta\left(\gamma_{1}^{-1} k \gamma_{1}\right)=-\delta(k) \in$ $A$. Hence $\gamma_{1}^{-1} k \gamma_{1} \in K_{A}$. As $H \triangleright K_{A}$ and $G_{B}=H \cup \gamma_{1}^{-1} H=H \cup \gamma_{1} H$, we have $G_{B} \triangleright K_{A}$.

Corollary 2.1. Let $A$ be a subgroup of $H_{1}(\widetilde{S}, \mathbb{Z})$ of index 3 . Then $K_{A}$ is a normal subgroup of $G_{B}$ such that $G_{B} / K_{A} \cong D_{6}$.

Proof. Choose $h \in H \backslash K_{A}$ such that $H=K_{A} \cup h K_{A} \cup h^{-1} K_{A}$. By Lemma 2.3. we have $\delta\left(\gamma_{1}^{-1} h \gamma_{1}\right)=\delta\left(h^{-1}\right)$. Hence we have $\gamma_{1}^{-1} h \gamma_{1} \in h^{-1} K_{A}$. This implies that $G_{B} / K_{A}$ is non-abelian.

2.4. Proof of Theorem 0.1. We keep our notation as before. Put $\varphi=\operatorname{pr}_{2} \circ$ $f \circ \mu$. We first note that $H_{1}(\widetilde{S}, \mathbb{Z}) \cong H_{1}\left(\left|\varphi^{-1}(0)\right|, \mathbb{Z}\right)$, where $|\bullet|$ denotes the underlying topological space of $\bullet$ by [3, Theorem 8.8, Chapter I] and [8]. We call the irreducible component of $\varphi^{-1}(0)$ coming from $\mathbb{P}^{1} \times\{0\}$ the main component. Our first observation is as follows:

Lemma 2.5. Let $\overline{\gamma_{2 g+2} \gamma_{2 g+3}}$ be the class of $\gamma_{2 g+2} \gamma_{2 g+3}$ in $H_{1}\left(\varphi^{-1}(0), \mathbb{Z}\right)$. Then

$$
H_{1}\left(\left|\varphi_{o}^{-1}(0)\right|, \mathbb{Z}\right) \oplus \mathbb{Z} \overline{\gamma_{2 g+2} \gamma_{2 g+3}} \cong H_{1}\left(\left|\varphi^{-1}(0)\right|, \mathbb{Z}\right) \text {. }
$$

Proof. We first note that the closure of $\varphi^{-1}\left(\mathbb{P}_{0}^{1} \backslash\left(B \cap \mathbb{P}_{0}^{1}\right)\right)$ in $\widetilde{S}$ gives either an irreducible curve $\Gamma_{0}$ or two irreducible curves $\Gamma_{0}^{+}$and $\Gamma_{0}^{-}$. We call them the essential component $(s)$ of $\varphi^{-1}(0)$. Note that $\Gamma_{0}^{ \pm} \cong \mathbb{P}^{1}$ since $f_{o}$ is a double cover.

By our construction, $\left|\varphi^{-1}(0)\right|$ is obtained by attaching a "pinched" handle as in Figure 1.

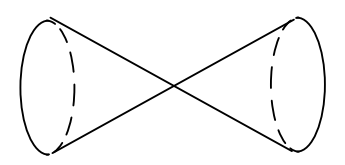

Figure 1. A pinched handle

Thus $\left|\varphi^{-1}(0)\right| \backslash$ a pinched handle is homotopic to $U_{1}, U_{1}$ is either $\left(\Gamma_{0}^{+} \backslash\right.$ a point $) \cup$ $\left(\Gamma_{0}^{-} \backslash\right.$ a point $) \cup$ other remaining components of $\varphi^{-1}(0)$ or $\Gamma_{0} \backslash$ two points $\cup$ other remaining components. 
Now let $U_{2}$ be the pinched handle body as in Figure 1. By applying the MayerVietoris exact sequence to $U_{1}$ and $U_{2}$, we have:

$$
\begin{aligned}
& H_{2}\left(U_{1} \cap U_{2}, \mathbb{Z}\right) \quad \rightarrow \quad H_{2}\left(U_{1}, \mathbb{Z}\right) \oplus H_{2}\left(U_{2}, \mathbb{Z}\right) \quad \rightarrow \quad H_{2}\left(\left|\varphi^{-1}(0)\right|, \mathbb{Z}\right) \quad \rightarrow \\
& H_{1}\left(U_{1} \cap U_{2}, \mathbb{Z}\right) \rightarrow H_{1}\left(U_{1}, \mathbb{Z}\right) \oplus H_{2}\left(U_{2}, \mathbb{Z}\right) \rightarrow H_{1}\left(\left|\varphi^{-1}(0)\right|, \mathbb{Z}\right) \rightarrow \\
& H_{0}\left(U_{1} \cap U_{2}, \mathbb{Z}\right) \rightarrow H_{0}\left(U_{1}, \mathbb{Z}\right) \oplus H_{0}\left(U_{2}, \mathbb{Z}\right) \rightarrow H_{0}\left(\left|\varphi^{-1}(0)\right|, \mathbb{Z}\right) \rightarrow 0 .
\end{aligned}
$$

Also by replacing $U_{2}$ by two disjoint discs, we have

$$
\begin{aligned}
& H_{2}\left(U_{1} \cap U_{2}, \mathbb{Z}\right) \rightarrow H_{2}\left(U_{1}, \mathbb{Z}\right) \oplus H_{2}\left(U_{2}, \mathbb{Z}\right) \rightarrow H_{2}\left(\left|\varphi_{o}^{-1}(0)\right|, \mathbb{Z}\right) \rightarrow \\
& H_{1}\left(U_{1} \cap U_{2}, \mathbb{Z}\right) \rightarrow H_{1}\left(U_{1}, \mathbb{Z}\right) \oplus H_{2}\left(U_{2}, \mathbb{Z}\right) \rightarrow H_{1}\left(\left|\varphi_{o}^{-1}(0)\right|, \mathbb{Z}\right) \rightarrow \\
& H_{0}\left(U_{1} \cap U_{2}, \mathbb{Z}\right) \rightarrow H_{0}\left(U_{1}, \mathbb{Z}\right) \oplus H_{0}\left(U_{2}, \mathbb{Z}\right) \rightarrow H_{0}\left(\left|\varphi_{o}^{-1}(0)\right|, \mathbb{Z}\right) \rightarrow 0 .
\end{aligned}
$$

For both exact sequences, we have that $U_{1} \cap U_{2}$ is homotopic to a disjoint union of two disjoint circles $S^{1}$. Hence we infer that

$$
H_{1}\left(\left|\varphi_{o}^{-1}(0)\right|, \mathbb{Z}\right) \cong \operatorname{Coker}\left(H_{1}\left(U_{1} \cap U_{2}, \mathbb{Z}\right) \rightarrow H_{1}\left(U_{1}, \mathbb{Z}\right)\right) .
$$

Hence we have

$$
H_{1}\left(\left|\varphi_{o}^{-1}(0)\right|, \mathbb{Z}\right) \oplus \mathbb{Z} \cong H_{1}\left(\left|\varphi^{-1}(0)\right|, \mathbb{Z}\right) .
$$

Since $\overline{\gamma_{i} \gamma_{i+1}}\left(i=1, \ldots, \gamma_{2 g+1}\right)$ generates $H_{1}\left(\left|\varphi_{o}^{-1}(0)\right|, \mathbb{Z}\right)$ and $\overline{\gamma_{2 g+3} \gamma_{2 g+4}}$ is 0 in $H_{1}\left(\left|\varphi^{-1}(0)\right|, \mathbb{Z}\right)$, we have our statement.

Put $A=H_{1}\left(\left|\varphi_{0}^{-1}(0)\right|, \mathbb{Z}\right) \oplus\left\langle\overline{\left(\gamma_{2 g+2} \gamma_{2 g+3}\right)^{3}}\right\rangle$, let $q: \widehat{X} \rightarrow \widetilde{S}$ be the cyclic triple cover corresponding to $K_{A}$ in $H$, and let $\nu: \widehat{X} \rightarrow \hat{S}$ be the Stein factorization of $\widehat{X} \rightarrow \widetilde{S} \rightarrow \mathbb{P}^{1} \times \Delta_{\epsilon}$. By Corollary 2.1, $\hat{S}$ is a $D_{6}$-cover, and we denote its covering morphism by $\hat{\pi}: \hat{S} \rightarrow \mathbb{P}^{1} \times \Delta_{\epsilon}$. By our construction, $\hat{\pi}^{-1}\left(\mathbb{P}^{1} \times\{0\}\right)$ satisfies the following conditions:

- $\hat{\pi}^{-1}\left(\mathbb{P}^{1} \times\{0\}\right)$ consists of three irreducible curves $F_{1}, F_{2}$ and $F_{3}$.

- We may assume that $\sigma^{*} F_{1}=F_{1}, \sigma^{*} F_{2}=F_{3}$ and $\tau^{*} F_{1}=F_{2}, \tau^{*} F_{2}=F_{3}$, where $\sigma$ and $\tau$ are the elements of $D_{6}$ as in $\S 1$.

- For each $i, \nu^{-1}\left(F_{i}\right)$ is isomorphic to $\varphi_{o}^{-1}(0)$. The involution induced by $\sigma$ acts on $\nu^{-1}\left(F_{i}\right)$ in the same way as the involution on $\widetilde{W}$ induced by the covering transformation of $f_{o}: W \rightarrow \mathbb{P}^{1} \times \Delta_{\epsilon}$ acts on $f_{o}^{-1}\left(\mathbb{P}^{1} \times\{0\}\right)$.

Let $X$ be the quotient surface of $\hat{S}$ by $\sigma$. By Lemma 1.1, $X$ is a non-Galois triple cover of $\mathbb{P}^{1} \times \Delta_{\epsilon}$ with branch locus $B$, and we denote its covering morphism by $\pi: X \rightarrow \mathbb{P}^{1} \times \Delta_{\epsilon}$. Let $\varphi_{X}: X \rightarrow \Delta_{\epsilon}$ be the induced fibration. Then we have

- $\varphi_{X}^{-1}(t)$ is a smooth curve of genus $g$ for $t \neq 0$ such that $\left.\pi\right|_{\varphi_{X}^{-1}(t)}: \varphi_{X}^{-1}(t) \rightarrow$ $\mathbb{P}^{1} \times\{t\}$ is a 3 -to- 1 morphism.

- $\varphi_{X}^{-1}(0)$ consists of two reduced components $G_{1}$ and $G_{2}$, where $G_{1}$ is the image of $F_{1}$ and $G_{2}$ is the image of both $F_{2}$ and $F_{3}$. Since $G_{1} \cong \mathbb{P}^{1}$ and $G_{1} G_{2}=1, G_{1}$ is an exceptional curve of the first kind. Also $G_{2}$ is isomorphic to $f_{o}^{-1}\left(\mathbb{P}^{1} \times\{0\}\right)$.

Let $x_{o}$ be a singular point of $X . y_{o}=\pi\left(x_{o}\right)$ is a singular point of $B_{1}$, and there exists a small neighborhood $U_{y_{o}}$ and $V_{x_{o}}$ of $y_{o}$ and $x_{o}$, respectively, such that $\pi\left(V_{x_{o}}\right)=U_{y_{o}}$ and $\left.\pi\right|_{V_{x_{o}}}: V_{x_{o}} \rightarrow U_{y_{o}}$ is a double cover. Since $\sigma$ just exchanges $F_{2}$ and $F_{3},\left.\pi\right|_{V_{x_{o}}}$ is locally isomorphic to $f_{o}$ over $U_{y_{o}}$. We now blow down $G_{1}$ and take the canonical resolution $\widetilde{X}$ of all singularities of $X$. Since $G_{1}$ is a vertical divisor, $\varphi_{X}$ induces another fibration $\varphi_{\widetilde{X}}: \widetilde{X} \rightarrow \Delta_{\epsilon}$, which gives the desired local trigonal fibration in Theorem 0.1 . 


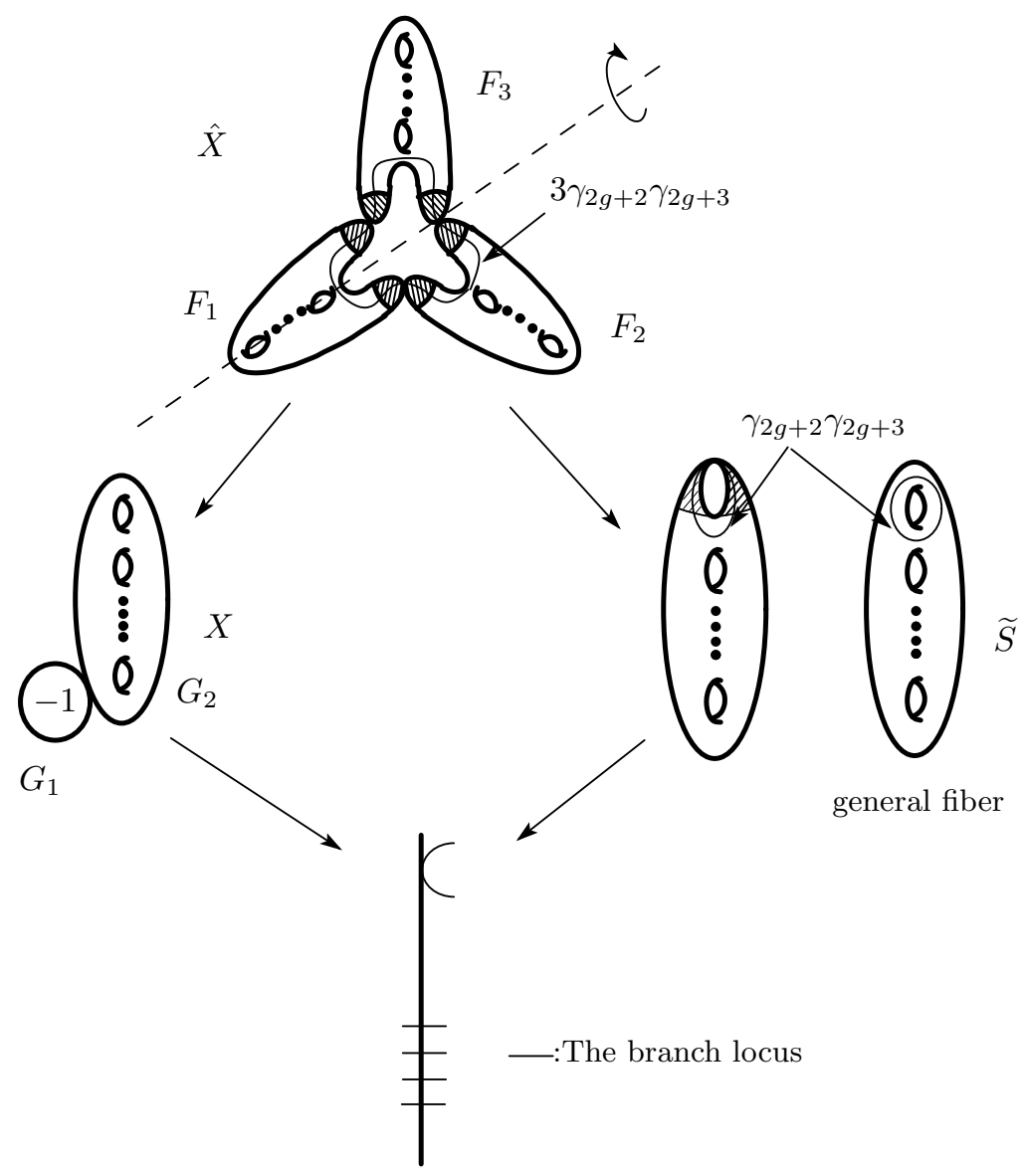

FiguRE 2

In the case when $B_{1}$ is smooth, the above observation can be explained in Figure 2 .

\section{AN EXAMPLE FOR THE NON-HORIZONTAL CASE}

In this section, we give a local trigonal fibration of genus 3 such that the central fiber is given as the one for a local hyperelliptic fibration of non-horizontal type.

Note that some of the local trigonal fibrations of non-horizontal type can be reduced to those of horizontal type by considering elementary transformations at $\mathbb{P}^{1} \times\{0\}$. Our example, given below, is the one which cannot be reduced to nonhorizontal type.

Example 3.1. Let $B_{1}$ be a reduced divisor on $\mathbb{P}^{1} \times \Delta_{\epsilon}$ given by

$$
B_{1}: t\left(x^{4}-t\right)\left(x^{4}+t\right)=0,
$$

where $x=X_{1} / X_{0},\left[X_{0}, X_{1}\right]$ being a homogeneous coordinate of $\mathbb{P}^{1}$ and $t$ is a coordinate of $\Delta_{\epsilon}$. Let $\varphi_{o}: \mathcal{C} \rightarrow \Delta_{\epsilon}$ be the local hyperelliptic fibration obtained as in the Introduction. $\varphi_{o}$ is not relatively minimal and let $\bar{\varphi}_{o}: \overline{\mathcal{C}} \rightarrow \Delta_{\epsilon}$ be its 
relatively minimal model. We denote its central fiber by $F_{0}$. The configuration of $F_{0}$ is

$$
F_{0}=4 E+2 C_{1}+2 C_{2},
$$

where $E$ is a curve of genus 1 and $C_{i}, i=1,2$, are smooth rational curves with $E^{2}=-1, C_{i}^{2}=-2, E C_{i}=1$ and $C_{1} C_{2}=0$.

We first note that $\bar{\varphi}_{o}: \overline{\mathcal{C}} \rightarrow \Delta_{\epsilon}$ in Example 3.1 is never obtained as the relatively minimal model of a local hyperelliptic fibration of horizontal type. In fact, suppose that there exists a local hyperelliptic fibration of horizontal type such that the relatively minimal model of $\varphi_{1}: \mathcal{C}^{\prime} \rightarrow \Delta_{\epsilon}$ is $\bar{\varphi}_{o}$. This means that $\mathcal{C}^{\prime}$ is obtained from $\overline{\mathcal{C}}$ by successive blowing-ups. Since $F_{0}$ has no reduced component, we infer that the central fiber of $\varphi_{1}$ also has no reduced component. On the other hand, the irreducible component of the central fiber of $\varphi_{1}$ arising from $\mathbb{P}^{1} \times\{0\}$ is reduced by taking a local section into account. This leads us to a contradiction.

Therefore we cannot apply Theorem 0.1 to obtain a local trigonal fibration with central fiber $F_{0}$ in Example 3.1. Nevertheless, there exists a local trigonal fibration with central fiber $F_{0}$. We end this section by constructing such an example explicitly.

Example 3.2. Let us start with a family of plane quartic curves as follows:

Let $\left[X_{0}, X_{1}, X_{2}\right]$ be the homogeneous coordinates of $\mathbb{P}^{2}$. Consider the surface $H_{0}$ of $\mathbb{P}^{2} \times \Delta_{\varepsilon}$ defined by

$$
\left(X_{1} X_{2}-X_{0}^{2}\right)^{2}+t^{8}\left(X_{1}^{4}-X_{2}^{4}\right)=0 .
$$

Let $\pi_{0}: H_{0} \rightarrow \Delta_{\varepsilon}$ be the morphism induced from the second projection $\mathbb{P}^{2} \times \Delta_{\varepsilon} \rightarrow$ $\Delta_{\varepsilon}$. We see that the fiber $\pi_{0}^{-1}(0)$ is the singular locus of $H_{0}$. Since the fibers $\pi_{0}^{-1}(t)$ $(t \neq 0)$ are non-singular plane curves of degree four, they are non-hyperelliptic, i.e., trigonal, curves of genus 3. We blow up $\mathbb{P}^{2} \times \Delta_{\varepsilon}$ along the ideal generated by $X_{1} X_{2}-X_{0}^{2}$ and $t^{4}$. Setting $Z_{0} t^{4}=X_{1} X_{2}-X_{0}^{2}$, we obtain the defining equation of the proper transformation $H_{1}$ of $H_{0}$ in the exceptional set as

$$
Z_{0}^{2}+\left(X_{1}^{4}-X_{2}^{4}\right)=0 .
$$

The family $\pi_{1}: H_{1} \rightarrow \Delta_{\varepsilon}$ is non-singular. Moreover, since the restriction $h: \pi_{1}^{-1}(0)$ $\rightarrow \pi_{0}^{-1}(0)$ of the morphism $H_{1} \rightarrow H_{0}$ is the double cover branched at eight points $X_{1} X_{2}-X_{0}^{2}=X_{1}^{4}-X_{2}^{4}=t=0$, the fiber $\pi_{1}^{-1}(0)$ is a hyperelliptic curve.

We now define the automorphism of $G: H_{0} \rightarrow H_{0}$ induced from the automorphism of $\mathbb{P}^{2} \times \Delta_{\varepsilon}$ as

$$
X_{0} \mapsto i X_{0}, \quad X_{1} \mapsto-X_{1}, \quad X_{2} \mapsto X_{2}, \quad t \mapsto i t,
$$

where $i=\sqrt{-1}$. The fixed points of $G$ are $P_{1}=[0,0,1]$ and $P_{2}=[0,1,0]$ on $\mathbb{P}^{2} \times\{0\}$. We can naturally define the automorphism $G^{\prime}: H_{1} \rightarrow H_{1}$ of $H_{1}$ induced from $G$. Note that $G^{\prime}$ acts on the coordinate $Z_{0}$ as $Z_{0} \mapsto-Z_{0}$. Consider the quotient $S$ of $H_{1}$ by the cyclic group $\left\langle G^{\prime}\right\rangle$ generated by $G^{\prime}$. We see that $\left(G^{\prime}\right)^{4}$ is the identity and $\left(G^{\prime}\right)^{2}$ has four fixed points on $\pi_{1}^{-1}(0)$ which are the inverse image of $P_{1}$ and $P_{2}$ by $h$. Since $G^{\prime}$ acts on the coordinate $Z_{0}$ as $Z_{0} \mapsto-Z_{0}$ and $G^{\prime}$ interchanges the two points of the inverse image of $P_{i}(i=1,2)$ by $h$, we see that the restriction $\left.G^{\prime}\right|_{\pi_{1}^{-1}(0)}$ of $G^{\prime}$ is the automorphism of order four and the quotient $\pi_{1}^{-1}(0) /\left\langle\left. G^{\prime}\right|_{\pi_{1}^{-1}(0)}\right\rangle$ is an elliptic curve. 
Thus, we see that the singular fiber $\phi^{-1}(0)$ of $\phi: S \rightarrow \Delta_{\varepsilon}$ is the elliptic curve with multiplicity four and $S$ has two rational double points of type $A_{1}$ on its singular

fiber. Then, the resolution $\psi: \widetilde{S} \rightarrow \Delta_{\varepsilon}$ of the family $\phi: S \rightarrow \Delta_{\varepsilon}$ has the singular fiber $F_{0}$ and its general fibers are non-hyperelliptic curves.

\section{REFERENCES}

[1] E. Artal, J. Carmona, and J.I. Cogolludo, Braid monodromy and topology of plane curves, Duke Math. J. 118 (2003), no. 2, 261-278. MR1980995 (2004k:14015)

[2] E. Artal Bartolo, J.-I. Cogolludo and H. Tokunaga, A survey on Zariski pairs, Adv. Stud. Pure Math., 50, Math. Soc. Japan, 2008, 1-100. MR2409555 (2009g:14030)

[3] W. Barth, C. Peters, and A. Van de Ven, Compact Complex Surfaces, Erg. der Math. und ihrer Grenz., A Series of Modern Surveys in Math., 3, vol. 4, Springer-Verlag, Berlin, 1984. MR749574 (86c:32026)

[4] Z. Chen and S.-L. Tan, Upper bounds on the slope of a genus 3 fibration, Contemp. Math., 400, Amer. Math. Soc., 2006. MR2222466 (2007j:14034)

[5] A. Dimca, Singularities and Topology of Hypersurfaces, Springer-Verlag, New York, 1992. MR.1194180 (94b:32058)

[6] A. Grothendieck, Revêtements étales et groupe fondamental, Lecture Notes in Math., 224, Springer-Verlag, Berlin, 1971. MR0354651 (50:7129)

[7] E. Horikawa, On deformation of quintic surfaces, Invent. Math. 31 (1975), 43-85.

[8] S. Lojasiewicz, Triangulation of semi-analytic sets, Ann. Sc. Norm. Sup. Pisa (3) 18 (1964), 449-474. MR0173265 (30:3478)

[9] M.V. Nori, Zariski's conjecture and related problems, Ann. Scient. Ec. Norm. Sup. 16 (1983), 305-344. MR732347 (86d:14027)

[10] M. Suzuki, Group Theory I, Grundlehren der mathematischen Wissenschaften, 247, SpringerVerlag, 1982. MR648772 (82k:20001c)

[11] S.-L. Tan, Triple covers on smooth algebraic varieties, Geometry and Nonlinear Partial Differential Equations, AMS/IP Studies in Adv. Math., 29, Amer. Math. Soc., and Intern. Press, 2002, 143-164. MR.1926442 (2003g:14020)

[12] H. Tokunaga, Triple coverings of algebraic surfaces according to the Cardano formula, J. of Math. Kyoto Univ. 31 (1991), 359-375. MR1121171 (92j:14047)

Department of Mathematical Sciences, Ritsumeikan University, 1-1-1 Noji Higashi KUSATSU 525-8577, SHIGA, JAPAN

Department of Mathematics and Information Sciences, Tokyo Metropolitan UniverSity, 1-1 Minami-Osawa, HachioJi-Shi 192-0397, Tokyo, Japan 